\title{
Can FES-augmented active cycling training improve locomotion in post-acute elderly stroke patients?
}

\author{
Elisabetta Peri (1), Emilia Ambrosini (1,2), Alessandra Pedrocchi (1), Giancarlo \\ Ferrigno (1), Claudia Nava (2), Valentina Longoni (2), Marco Monticone (2), Simona \\ Ferrante (1) \\ (1) Nearlab, Department of Electronics Informatics and Bioengineering, Politecnico di \\ Milano, Milano, Italy; (2) Physical Medicine and Rehabilitation Unit, Scientific Institute of \\ Lissone, S. Maugeri Foundation, IRCCS, Lissone, Italy.
}

This article is distributed under the terms of the Creative Commons Attribution Noncommercial License (CC BY-NC 4.0) which permits any noncommercial use, distribution, and reproduction in any medium, provided the original author(s) and source are credited.

\begin{abstract}
Recent studies advocated the use of active cycling coupled with functional electrical stimulation to induce neuroplasticity and enhance functional improvements in stroke adult patients. The aim of this work was to evaluate whether the benefits induced by such a treatment are superior to standard physiotherapy. A single-blinded randomized controlled trial has been performed on post-acute elderly stroke patients. Patients underwent FES-augmented cycling training combined with voluntary pedaling or standard physiotherapy. The intervention consisted of fifteen 30-minutes sessions carried out within 3 weeks. Patients were evaluated before and after training, through functional scales, gait analysis and a voluntary pedaling test. Results were compared with an age-matched healthy group. Sixteen patients completed the training. After treatment, a general improvement of all clinical scales was obtained for both groups. Only the mechanical efficiency highlighted a group effect in favor of the experimental group. Although a group effect was not found for any other cycling or gait parameters, the experimental group showed a higher percentage of change with respect to the control group (e.g. the gait velocity was improved of $35.4 \%$ and $25.4 \%$ respectively, and its variation over time was higher than minimal clinical difference for the experimental group only). This trend suggests that differences in terms of motor recovery between the two groups may be achieved increasing the training dose. In conclusion, this study, although preliminary, showed that FESaugmented active cycling training seems to be effective in improving cycling and walking ability in post-acute elderly stroke patients. A higher sample size is required to confirm results. Key Words: active cycling coupled with functional electrical stimulation, single-blinded randomized controlled
\end{abstract}

Eur J Transl Myol 26 (3): 187-192

Stroke is one of the most common neurological disorder affecting 15 million people worldwide, of whom one third remains permanently disabled, with high social and economical costs ${ }^{1}$. The induced neurological deficit results in an impaired motor control of the hemisoma opposite to the cerebral damage, affecting the patients' locomotion ability and their quality of life.

A rehabilitative intervention early after injuries is crucial to maximize the possibility of recovery as it could enhance the cortical reorganization ${ }^{2}$ thus facilitating the relearning of the compromised movements.

Functional Electrical Stimulation (FES) is a rehabilitative tool which induces an artificial muscular contraction through a train of electrical stimuli, with the aim to achieve a functional movement. Its use in stroke population has been investigated by a recent review study showing both short and long terms effects ${ }^{3}$. The carry-over effect could be explained considering that, thanks to the augmented proprioception and the induced afferent volleys, FES could play a role in the neuroplasticity both at cortical and spinal level. These effects are enhanced if FES is synchronized with a volitional effort ${ }^{4,5}$.

A safe and widely accessible way to achieve FES benefits is the association with a cycling movement (FES cycling). The cycling movement and the locomotion share some important characteristics 6: they are both cyclical, require alternating flexion and 
extension of the lower limb joints and the coordination of agonist and antagonist muscles. A pedaling-based training may thus lead to an improvement of gait ability. Moreover, FES cycling is repetitive and goal-oriented, aspects which are recognized to be key factors in promoting the neuroplasticity and, thus, a long-term recovery. Studies on post-acute stroke ${ }^{7,8,9}$ show that FES cycling could improve significantly the motor functions and fasten the recovery of the locomotion ability with respect to standard care. Some carry-over effects were also observed as the improvements were maintained in a 3-months follow up. A limiting factor to these studies was that they did not exploit the descendant volley synchronized to the FES-induced movement, thus limiting the possibility of neuroplasticity.

The aim of this study was to demonstrate whether a FES augmented voluntary cycling treatment is superior to usual care. To reach this aim a randomized controlled trial (RCT) was designed and the preliminary results obtained on 16 patients are here reported.

\section{Material and Methods}

\section{Study design}

A single-blinded (blind assessors) randomized controlled trial was carried out to compare FESaugmented active cycling therapy and standard care in terms of locomotion recovery. A blocking randomization (random permuted blocks) was used to create the allocation list. This method assures allocation concealments and an equal size of the two groups. Inclusion criteria were: patients at their first stroke in post-acute phase (less than 6-months from stroke occurrence), age higher than 18, Modified Ashworth Scale $<=2$, lack of articular limitation at hip, knee and ankle, suficient cognitive ability (Mini Mental State Examination $>=25)$. The research protocol was approved by the ethical committee of the Fondazione Salvatore Maugeri rehabilitation center.

The gait speed was considered as the primary end-point to evaluate the sample size. Considering a statistical power of $80 \%$, a $5 \%$ of type I error, and a betweengroup difference of $0.16 \mathrm{~m} / \mathrm{s}$ with a standard deviation of $0.22 \mathrm{~m} / \mathrm{s}$, a sample size of 60 patients was computed.

\section{Intervention}

Participants were randomly allocated to an experimental or a control group. Both of them underwent 75 minutes of training for 15 days within 3 weeks.

The experimental program involved 25 minutes of FESaugmented active cycling and 50 minutes of standard physiotherapy. During the cycling training, FES was delivered to the quadriceps, hamstrings, tibialis anterior and gastrocnemius lateralis muscles of both legs. The paretic leg muscles received stimulation at the maximum intensity tolerated by the patient, while the not-affected leg muscles was stimulated with an intensity intended to produce just a visible contraction.
Each FES session consisted of 2.5 minutes of passive pedaling on a motorized cycle-ergometer (warm-up phase), 2.5 minutes of passive FES, 15 minutes of FES synchronized with voluntary pedaling, 2.5 minutes of passive FES, and 2.5 minutes of passive pedaling (warm-down phase). During the training, the patients were provided with a visual feedback of the tangential force to help them perform a symmetrical task. A MOTOmed Viva2 ergometer (Reck GmbH, DE) customized with sensors measuring the tangential and the radial forces at the two pedals (Powerforce ${ }^{\mathrm{TM}}$, Radlabor GmbH, DE), and an 8-channel currentcontrolled stimulator (RehaMove $2^{\mathrm{TM}}$ Hasomed $\mathrm{GmbH}$, $\mathrm{DE})$ were used for the intervention.

The control group was involved in 75 minutes of standard physiotherapy (strength and stretching exercises, gait training, stairs, hand rehabilitation, etc).

\section{Assessment tests and data analysis}

Before (T1) and after (T2) the training, the patients were evaluated by means of functional scales, a walking test and a cycling test.

The functional scales consisted of the 6-minutes walking test (6MWT), which assessed the endurance during walking, and the Functional Independence Measure (FIM) to evaluate the physical and cognitive (FIMmot, FIMcog) level of disability.

Some spatio-temporal gait parameters were also considered. In particular, the gait speed (v) and the double support time (Tds) were selected. Data acquisition was carried out asking patients to walk 3 times on a sensorized mat (GAITRite $\AA$, CIR System Inc, USA).

Finally, a cycling test was performed. The same sensorized cycle ergometer described in the previous paragraph was used and the patients were asked to perform 4 tests at different velocities (20, 30, 40 and 50 revolutions per minute or RMP). Each test consisted of 1 minute of passive pedaling (i.e. the ergometer provided the whole movement) followed by 2 minutes of active pedaling at a customized resistance.

For each revolution, the tangential force profile was plotted as function of the crank angle. The contribution due to the active pedaling was then evaluated by subtracting the mean values obtained during the passive phase. For each target cadence, the work produced by the paretic (Wp) and the healthy leg (Wh) were computed as the integral of the active force profile and averaged among all revolutions.

The unbalance U\% between the works produced by the two legs was calculated then as in Eq. 1.

$$
U \%=\frac{|W p-W h|}{W p+W h} \%
$$

Furthermore, an Area Symmetry Index (ASI) was evaluated as described by ${ }^{10}$ and reported in Eq. 2 .

$$
A S I=1-\frac{\sum_{n=1}^{360}\left|F_{p}(n)-F_{h}(n)\right|}{\sum_{n=1}^{360} F_{p}(n)+\sum_{n=1}^{360} F_{h}(n)}
$$




\section{Volitional FES-cycling to improve locomotion in post-acute elderly stroke patients}

Where Fp and Fh represent the tangential active force profile of the paretic and healthy leg, respectively.

Finally, a mechanical efficiency index (EI) was also computed as the ratio between the mean tangential component of the force (Ftg) and the mean total force (Ftot, the resultant of the tangential and radial force), as in Eq. 3. A IE value of $0.34 \pm 0.04$ is obtained by elderly healthy subjects during recumbent cycling (see Table II).

$$
E I=\frac{\sum_{i=1}^{360} F \operatorname{tg}(i)}{\sum_{i=1}^{360} F \operatorname{tot}(i)}
$$

In order to have indexes comprehensive of the overall performance of the subjects at different velocities, for all the parameters the mean values at the four different velocities were computed.

The cycling test was delivered to an age-matched control group $(\mathrm{N}=12$, age $>60$ years $)$ to have reference values.

\section{Statistics}

The normality of each variable has been verified using the Lillietest and, according to this result, a parametric (normal data) or non-parametric analysis (non-normal data) was performed and the corresponding values are presented as mean (standard deviation) or as median [inter-quartile].

First, the difference between the experimental and control group at baseline was evaluated (unpaired t-test or Mann-Whitney U-test, for normal or non-normal data, respectively).

For each of the outcome measures, a linear mixed model analyses for repeated measures $(\mathrm{p}<0.05)$ was carried out with group and time entered as fixed effects and the outcome measures entered as dependent variables. The crossover effect of time by group was entered as an interaction term. For the non-parametric data, a preliminary aligned rank transform procedure was required before applying the linear mixed model. The ARTool software was used ${ }^{11}$

The comparison with the healthy population was performed for the cycling parameters with a non parametric Mann-Whitney U-test. The statistical analysis was performed with IBM SPSS Statistics v23 software.

\section{Results}

Up to now, 16 patients, 8 for each group, were recruited and completed the study. Participants' details are in Table I. No significant differences at baseline were found for any of the outcome measures considered. The results are in Table II.

\section{Functional scales}

The 6MWT showed a significant improvement over time for both groups. The performance always doubled over time. Both groups obtained a significant
Table 1. Patient's details at baseline

\begin{tabular}{lcc}
\hline & $\begin{array}{c}\text { Experimental } \\
\text { Group }\end{array}$ & $\begin{array}{c}\text { Control } \\
\text { Group }\end{array}$ \\
\hline Age $^{\mathrm{a}}$, years & $71.8 \pm 12.9$ & $76.4 \pm 8.7$ \\
\hline Gender (F/M) & $6 / 2$ & $3 / 5$ \\
\hline Affected side (R/L) & $6 / 2$ & $1 / 7$ \\
\hline Days post stroke & $14.4 \pm 2.7$ & $16.0 \pm 5.5$ \\
\hline Motricity Index (MI) & $76.13 \pm 9.52$ & $64.14 \pm 19.00$ \\
\hline & \multicolumn{2}{c}{ a, mean \pm standard deviation }
\end{tabular}

improvement over time of the FIM (45.2\% and $47.9 \%$ for experimental and control group, respectively).

Considering the subscales, FIMmot obtained a significant improvement over time. Differently, the cognitive evaluation FIMcog did not obtain any significant difference over time and between the two groups.

\section{Gait analysis}

The gait speed significantly increases over time for both experimental and control group (35.4\% and $24.3 \%$ respectively). The double support time, Tds, was significantly reduced for both groups $(33.8 \%$ and $23.0 \%$ respectively), towards the normality ranges.

\section{Cycling test}

Figure 1 shows the tangential force produced at $\mathrm{T} 1$ and T2 during pedaling at 30 RPM by the affected (left panels) and unaffected leg (right panels) of both experimental (upper panels) and control (lower panels) groups, in comparison with the results obtained by the healthy subjects (green line and area). Both groups at T2 highlighted a higher peak value during knee extension for the affected leg. The experimental

group showed an improving trend during both knee flexion and extension phases while the control group during knee flexion maintained the performance of T2 very similar to $\mathrm{T} 1$.

The total amount of work produced during the whole movement for the affected side (Wp) significantly changed with time $(30.5 \%$ and $11.1 \%$ for the experimental and control group, respectively). Both groups were significantly different from the healthy population at $\mathrm{T} 1$ but only the control group maintained this difference at T2. However, nor the difference between groups or the interaction between time and group were significant.

The symmetry during cycling (Unbalance variation of $20.5 \%$ and $3.7 \%$ for the experimental and control group; ASI variation of $10.5 \%$ and $5.8 \%$ for the experimental and control group) was significantly improved for both groups over time but maintained values significantly different from the healthy population.

The mechanical efficiency IE of the experimental group was significantly higher after training $(11.3 \%)$ while the control group obtained a lower variation (8.5\%). However, the experimental group showed values similar 
Table 2. Results obtained with functional scales, gait analysis and pedalling test. Mean (standard deviation) and median [interquartile] are reported accordingly to the normality test.

* if u-Mann Whitney $p<0.05$, bold values if mixed linear model $p<0.05$

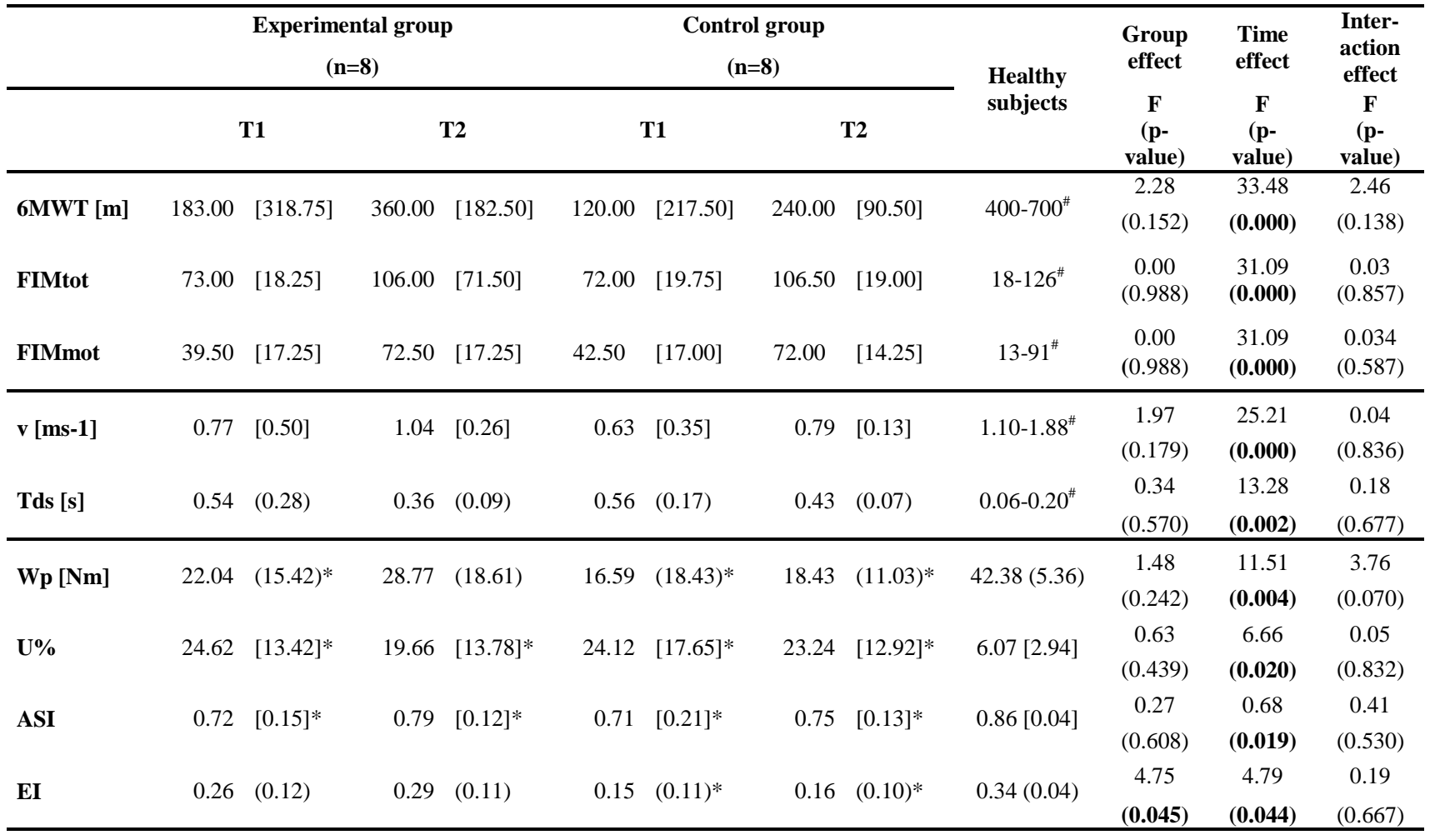

* indicates a significant difference with respect to the healthy group \# Ranges from literature are reported

to the healthy subjects already at baseline, maintained at $\mathrm{T} 2$, while the control group obtained values different for the healthy population during both evaluations.

\section{Discussion}

A randomized control trial has been carried out on 16 post-acute stroke adult patients. The experimental group performed a 15-sessions FES-augmented active cycling treatment and standard physiotherapy while the control group received an equal dose of standard physiotherapy only. The results showed an improving trend for all the outcome measures considered, with few significant differences between the experimental and the control group.

The functional scales allowed to observe significant improvements over time. The increase in terms of FIM was due to a significantly enhanced motor independence as assessed by FIMmot. Indeed, FIMmot increased of 33.0 and 29.5 points for the experimental and control group, respectively, both higher than the minimum clinical difference $\left(\mathrm{MCD}=17\right.$ points) ${ }^{12}$. This supports that motor abilities in daily life activity were improved by the treatment.

An analysis of locomotion-related measures has also been performed. The distance walked during the 6MWT increased far over the MCD $(50 \mathrm{~m})$ reported in literature for stroke patients ${ }^{13}$ suggesting an improved endurance during walking for both groups.
The gait speed also increased significantly no matter from the group but only the experimental group obtained a median improvement higher than the MCD for the gait speed $(0.16 \mathrm{~m} / \mathrm{s})^{14}$. Moreover, the double support time was significantly reduced over time, towards a less impaired gesture.

Concerning the cycling test, some improvements were shown over time for all the indexes considered. After the treatment, the patients were able to produce a higher work with the paretic leg, with a more symmetrical and efficient pattern. Previous findings by our gorup ${ }^{7}$ showed that variation in terms of the paretic work and the unbalance obtained with a 20 sessions FES-cycling training had a mean percentage variation over time of $105.6 \%$ and $20.9 \%$, respectively. While the unbalance change was comparable with what described above, the higher change of the paretic work could be due to a higher dose of training (20 sessions) that the patients received in the previous study with respect to the present study (15 sessions). However, the different result may also be due to the younger and more impaired patients (mean age $59 \pm 10, \quad \mathrm{MI}=38 \pm 55$ ) analyzed by the previous study with respect to this study (see Table I).

The group factor seems to be not relevant except for the mechanical efficiency index (IE) for which the experimental group had a wider recovery. However, they also had a non-significant higher baseline that may 

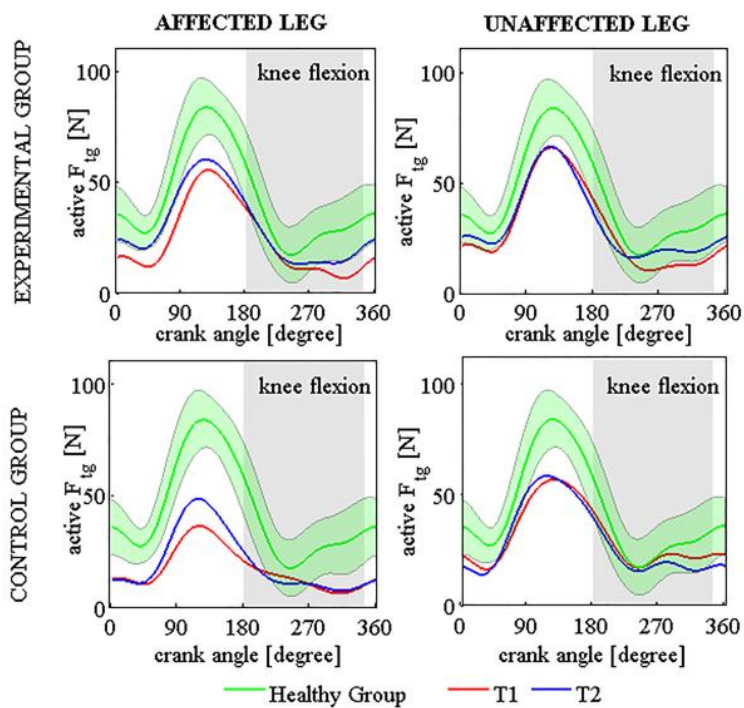

Fig 1. Force produced during pedaling (Ftg) at 30 RPM by affected and unaffected limb for both experimental and control group. Knee flexion phase is highlited as gray area. Red and blue lines are the mean values at $T 1$ and $T 2$ respectively for the patients, green line and area are mean and standard deviation results for healthy population.

affect this result. Although no other significance in terms of group factor was found for the cycling indexes, a deeper analysis shows that a trend of wider improvement seems to be obtained by the experimental group that directly trained the task during the rehabilitative treatment. In fact, the experimental group had a wider percentage of improvement for all of the cycling-related parameters and, moreover, its median Wp after the treatment (T2) was comparable with what obtained by the healthy subjects.

A wider trend of improvement for the experimental group was also obtained on locomotion-related measures. Indeed, only the experimental group showed a gait speed change higher than the MCD.

The preliminary results here presented suggest that a 15sessions of FES-augmented active training may not be enough to observe a different evolution of the recovery between the experimental and the control group. It should be considered that patients were recruited only few days after stroke (mean of 14.4 and 16.0 days from the stroke for the experimental and the control group, respectively) and thus part of the time effect can be ascribed also to the spontaneous recovery which occurred soon after stroke. To evaluate more accurately the group effect, the a-priori computed sample size of 60 patients should be achieved. Finally, the results should be enriched considering the long-term effects of the training.

In conclusion, a single-blinded randomized control trial has been performed and some promising results have been obtained. A higher therapeutic dose and a larger sample size may be required to lead to conclusive results.

\section{Acknowledgments}

The authors would like to acknowledge the doctors, the therapists and the participants for their support to the study.

Research was supported by the Italian Ministry of Healthy (Grant no.: GR-2010-2312228, title "Fall prevention and locomotion recovery in post-stroke patients: a multi-modal training towards a more autonomous daily life").

\section{Conflict of Interest}

The authors declare no potential conflict of interests.

\section{Corresponding Author}

Elisabetta Peri, Nearlab, Department of Electronics Informatics and Bioengineering, Politecnico di Milano, Milano, Italy, Email: elisabetta.peri@polimi.it

\section{E-mails of coAuthors}

Emilia Ambrosini - emilia.ambrosini@polimi.it Alessandra Pedrocchi - alessandra.pedrocchi@polimi.it Giancarlo Ferrigno - giancarlo.ferrigno@ polimi.it Claudia Nava - navacla89@gmail.com

Valentina Longoni - valentina.longoni@libero.it Marco Monticone - marco.monticone@fsm.it Simona Ferrante - simona.ferrante@polimi.it

\section{References}

1. Ravindranath V, Dang HM, Goya RG, et al. Regional research priorities in brain and nervous system disorders. Nature 2015 ;527(7578):S198S206.

2. Xu Y, Hou QH, Russell SD, et al. Neuroplasticity in post-stroke gait recovery and noninvasive brain stimulation. Neural Regen Res 2015;10: 2072.

3. Kafri M, Laufer Y. Therapeutic effects of functional electrical stimulation on gait in individuals post-stroke. Ann Biomed Eng 2015;43:451-66.

4. Barsi GI, Popovic DB, Tarkka IM, Sinkjær T, Grey MJ. Cortical excitability changes following grasping exercise augmented with electrical stimulation. Exp Brain Res 2008;191:57-66.

5. Rushton DN. Functional electrical stimulation and rehabilitation - an hypothesis. Med Eng Phys 2003;25:75-8.

6. Burnfield JM, Shu Y, Buster T, Taylor A. Similarity of joint kinematics and muscle demands between elliptical training and walking: implications for practice. Phys Ther 2010;90:289 305.

7. Ambrosini E, Ferrante S, Pedrocchi A, Ferrigno G, Molteni F. Cycling induced by electrical stimulation improves motor recovery in postacute 


\section{Volitional FES-cycling to improve locomotion in post-acute elderly stroke patients}

Eur J Transl Myol 26 (3): 187-192

hemiparetic patients a randomized controlled trial. Stroke, 2011;42:1068-73.

8. Ambrosini E, Ferrante S, Pedrocchi A, Ferrigno G, Molteni V. Cycling induced by electrical stimulation improves muscle activation and symmetry during pedaling in hemiparetic patients. Neural Systems and Rehabilitation Engineering, IEEE Transactions 2012;20:320-30.

9. Ferrante S, Pedrocchi A, Ferrigno G, Molteni F. Cycling induced by functional electrical stimulation improves the muscular strength and the motor control of individuals with post-acute stroke. Eur J Phys Rehabil Med 2008;44:159-67.

10. Lin SI, Lo CC, Lin PY, et al. Biomechanical assessments of the effect of visual feedback on cycling for patients with stroke. J Electromyogr Kinesiol2012;22:582-8.
11. Wobbrock JO, Findlater L, Gergle D, et al. The aligned rank transform for nonparametric factorial analyses using only anova procedures. In: Proceedings of the SIGCHI Conference on Human Factors in Computing Systems.ACM, 2011;143-6.

12. Beninato M, Gill-Body KM, Salles D, et al. Determination of the minimal clinically important difference in the FIM instrument in patients with stroke. Arch Phys Med Rehabil 2006;87:32-9.

13. Perera S, Mody S, Woodman R, Studenski S. Meaningful change and responsiveness in common physical performance measures in older adults. J Am Geriatr Soc 2006;54:743-9.

14. Tilson JK, Sullivan KJ, Cen SY, et al. Meaningful gait speed improvement during the first 60 days poststroke: minimal clinically important difference. Phys Ther 2010;90:196-208. 